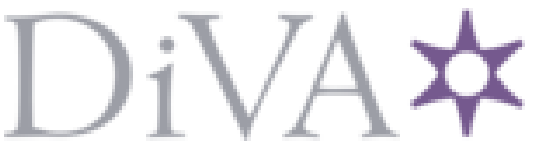

http://www.diva-portal.org

Preprint

This is the submitted version of a paper published in The international journal of pavement engineering.

Citation for the original published paper (version of record):

Svenson, K., McRobbie, S., Alam, M. (2019)

Detecting road pavement deterioration with finite mixture models

The international journal of pavement engineering, 20(4): 458-465

https://doi.org/10.1080/10298436.2017.1309193

Access to the published version may require subscription.

N.B. When citing this work, cite the original published paper.

Permanent link to this version:

http://urn.kb.se/resolve?urn=urn:nbn:se:du-24767 


\title{
Detecting Road Pavement Deterioration with Finite Mixture Models
}

\author{
Kristin Svenson, Stuart McRobbie, Moudud Alam
}

\begin{abstract}
Budget restrictions often limits the number of possible maintenance activities in a road network each year. To effectively allocate resources, the rate of road pavement deterioration is of great importance. If two maintenance candidate segments have equivalent condition, it is reasonable to maintain the segment with the highest deterioration rate first. To identify such segments, finite mixture models were applied to road condition data from a part of the M4 highway in England. Assuming that data originates from two different normal distributions - a "change" distribution and an "unchanged" distribution - all road segments were classified into either of these groups. Comparisons with known measurment errors and maintenance records showed that segments in the unchanged group were similar to segments having a stationary road condition, and that the change group consisted of segments with a rapid deterioration, improvement in condition due to previous maintenance or unusual mesurement errors. Finite mixture models can effectively find segments whose road condition have changed considerably. Together with additional information from maintenance records it is possible to identify segments with the most rapid deterioration rate, and hence make more efficient maintenance decisions.
\end{abstract}

Keywords: Finite Mixture Models, Pavement Deterioration, Road Maintenance

\section{Introduction}

There is a constant challenge in optimizing the effectiveness of maintenance planning. Modern database systems can store road condition data for short segments of an entire road network, hence making it possible to analyze micro data for macro scale applications. However, it is not realistic to manually examine and process datasets of such magnitudes, and the use of statistical models that can display relevant information become increasingly important.

Probabilistic modeling of pavement deterioration is a well explored research topic. Some examples are Lethanh and Adey (2012), who use exponential hidden Markov models to model deterioration when road condition data is sparse. Hong and Prozzi (2006) apply a Bayesian approach and find it successful in finding parameter distributions for their pavement performance model. However, when finding the actual maintenance candidate objects, the maintenance planner has to look at the condition data and determine which sections should be maintained instantly, and which can wait to the next budget period. In this decision, the deterioration that have occurred so far sometimes matters more than predicted future deterioration. Ideally, starting at the same condition, sections that have a higher deterioration rate should be maintained before those that deteriorate slower. Identifying the road segments that have deteriorated most in the previous year(s) can help deciding how maintenance candidate objects should be prioritized.

In order to find the segments with the highest deterioration rate, a way of clustering segments based on changes measured in road condition data is needed. Clustering methods is a wide topic 
in both statistics and machine learning. Algorithms for clustering include connectivity based clustering which uses distance as a mean of clutering (see e.g. Ward Jr, 1963; Sibson, 1973; Defays, 1977), k-means clustering which assigns an object to the nearest defined cluster center (see e.g. Steinhaus, 1956; Hartigan and Wong, 1979), and distribution-based clustering which distinguish clusters in data based on probabilistic models. The distribution-based models can capture correlations and dependencies between variables in a way that machine learning models often cannot. They are therefore a good choice when the theoretical foundation of the model is of importance, as well as the actual clustering.

In the class of distribution-based clustering methods, finite mixture models are common (McLachlan and Basford, 1988). Finite mixture models have been used by e.g. Park and Lord (2009) who combined mixtures of two different distributions to find sub-populations in motor vehicle crash data from Toronto, Canada. It is common in settings such as biocemistry where mixture models is used to model protein evolution (Lee et al., 2008), in genetics where it is used to detect heterogeneity in gene sequences (Pagel and Meade, 2004), and in medicine where heart rate variability is modelled with gaussian mixture models (Daeyoung and Lindsay, 2015). Finite mixture models can also be valuable for their flexibility when modelling unknown distributions, as proposed by Laitila and Karlsson (2014).

Assuming that road segments with a higher deterioration rate come from a wider distribution - a "change" distribution - and that segments with constant deterioration come from a more narrow distribution - an "unchanged" distribution - finite mixture models can be used to classify segments into either of these groups. In literature, this type of mixture is described as an outlier density: two normal distributions with similar mean but different variances (McLachlan and Peel, 2000, p. 14). By applying a multivariate distribution of several road condition variables, as much information as possible is used to distinguish between real changes and random errors.

The aim of this paper is to evaluate if finite mixture models can help identify road segments that have a higher deterioration rate by clustering segments into a "change" and an "unchanged" group.

\section{Methods and Materials}

\subsection{Data material}

The data material used in this study comes from the Traffic-speed Condition Surveys (TRACS), which is performed yearly on the road network maintained by Highways England. The finite mixture models are fitted to road condition measurement data from January 2013 to January 2015, measured on parts of the M4 highway and it's junction with the A34 in west Berkshire. Only lane one, the left most lane in each direction, is chosen. The total length of this road is 88.24 kilometers divided into 10 meter segments.

Several variables are measured within TRACS, including coordinates, longitudinal profile, transverse profile, and texture. The accuracy of the measurements is very important to capture real deterioration. Each measurement have a random error, but systematic errors can also occur, e.g. because of the calibration of the survey vehicle. To minimize possible sources of systematic errors, only surveys from one single contract period is used. Within a contract period, one company is contracted to do the TRACS measurements with equipment that is calibrated according to the same criteria each year. Between contract periods, different companies using vehicles with different calibration methods can cause systematic measurement errors. In England, a contract period is typically three years.

Another source of systematic errors is alignment. If the segments are not correctly aligned between each measurement occasion, the potential change that is found could simply occur 
because different sites are compared. Before the finite mixture models are fitted to the data, it has been through a process of alignment using GPS data in the first step, and image based alignment in the second step. The GPS alignment is a coarse method, while the image based assessment ensures that the alignment works also on a micro scale such as 10 meter segments (source).

As a reference for the change group segments, maintenance data from the Highways Agency Pavement Management System (HAPMS) is used. This data consists of sections where some part(s) of the section have been maintained. Exact GPS locations of the maintained sites are not available. The maintenance data is used to identify sections that have been maintained between measurement occasions. Segments in the change group within a section that have been maintained have most likely changed because of maintenance, and not deterioration.

\subsubsection{Road condition variables}

Eleven road condition variables, measuring different aspects of road deterioration, are chosen as the basis for the clustering. The transverse profile is represented by rutting measured in the left and right wheelpath respectively. The longitudinal profile is measured using enhanced longitudinal profile variance (ELPV) measured on the near side (NS) and off side (OS) of the road on maximum 3,10 and 30 meter wavelenghts. The texture is measured by the mean values of Root Mean Square Texture (RMST) measured on the near side, middle and off side of the road.

Table 1: Road condition variables

\begin{tabular}{|l|cc|cc|cc|}
\hline \multicolumn{2}{|c|}{2013} & \multicolumn{2}{c|}{2014} & \multicolumn{2}{c|}{2015} \\
\hline Variable & $\begin{array}{c}\text { Mean } \\
(\mathrm{mm})\end{array}$ & $\begin{array}{c}\text { Std } \\
(\mathrm{mm})\end{array}$ & $\begin{array}{c}\text { Mean } \\
(\mathrm{mm})\end{array}$ & $\begin{array}{c}\text { Std } \\
(\mathrm{mm})\end{array}$ & $\begin{array}{c}\text { Mean } \\
(\mathrm{mm})\end{array}$ & $\begin{array}{c}\text { Std } \\
(\mathrm{mm})\end{array}$ \\
\hline Left rutting & 3.3 & 2.3 & 3.3 & 2.1 & 3.3 & 1.9 \\
Right rutting & 3.5 & 2.6 & 3.5 & 2.4 & 2.9 & 2.3 \\
ELPV 3 m NS & 0.2 & 0.3 & 0.2 & 0.5 & 0.2 & 0.3 \\
ELPV 3 m OS & 0.2 & 0.5 & 0.2 & 0.5 & 0.1 & 0.2 \\
ELPV 10 m NS & 1.0 & 1.5 & 0.8 & 1.4 & 0.8 & 1.3 \\
ELPV 10 m OS & 1.0 & 1.8 & 0.8 & 1.7 & 0.8 & 1.3 \\
ELPV 30 m NS & 8.7 & 10.6 & 5.7 & 6.9 & 5.7 & 6.5 \\
ELPV 30 m OS & 8.3 & 10.3 & 5.3 & 6.9 & 5.1 & 6.4 \\
RMST Mean Middle & 1.1 & 0.2 & 1.0 & 0.3 & 1.0 & 0.2 \\
RMST Mean NS & 0.8 & 0.2 & 0.8 & 0.2 & 0.8 & 0.2 \\
RMST Mean OS & 0.8 & 0.2 & 0.8 & 0.2 & 0.8 & 0.2 \\
\hline
\end{tabular}

To be able to find real change in the road condition variables the extent of random measurement errors are of interest. These are evaluated by using measurements from a test site, where the accuracy of the survey vehicle is tested. The vehicle has surveyed all variables of a 2.2 kilometer long road section (divided into 10 meter segments) on five occasions in January 2013: the $1^{\text {st }}, 12^{\text {th }}, 17^{\text {th }}, 18^{\text {th }}$ and $25^{\text {th }}$ and no variable is expected to show any measurable change in that time. The difference in measurements between each occasion is calculated and plotted in histograms (Figure 1). For rutting, almost all measurement errors lie within $\pm 2 \mathrm{~mm}$ and 90 percent of the error lies within approximately $\pm 0.96 \mathrm{~mm}$ for left rutting and $\pm 1.1 \mathrm{~mm}$ for right rutting. $3 \mathrm{~m}$ ELPV has 90 percent of the measurement errors within approximately $\pm 0.37 \mathrm{~mm}$ for near side measurements and $\pm 0.27 \mathrm{~mm}$ for off side measurements, $10 \mathrm{~m}$ ELPV within $\pm 1 \mathrm{~mm}$ for both near side and off side and $30 \mathrm{~m}$ ELPV within $\pm 4.5 \mathrm{~mm}$ for both sides. 
For RMST, 90 percent of the measurement errors are within approximately $\pm 0.14 \mathrm{~mm}$ for near side, $\pm 0.2 \mathrm{~mm}$ for middle and $\pm 0.16 \mathrm{~mm}$ for off side.
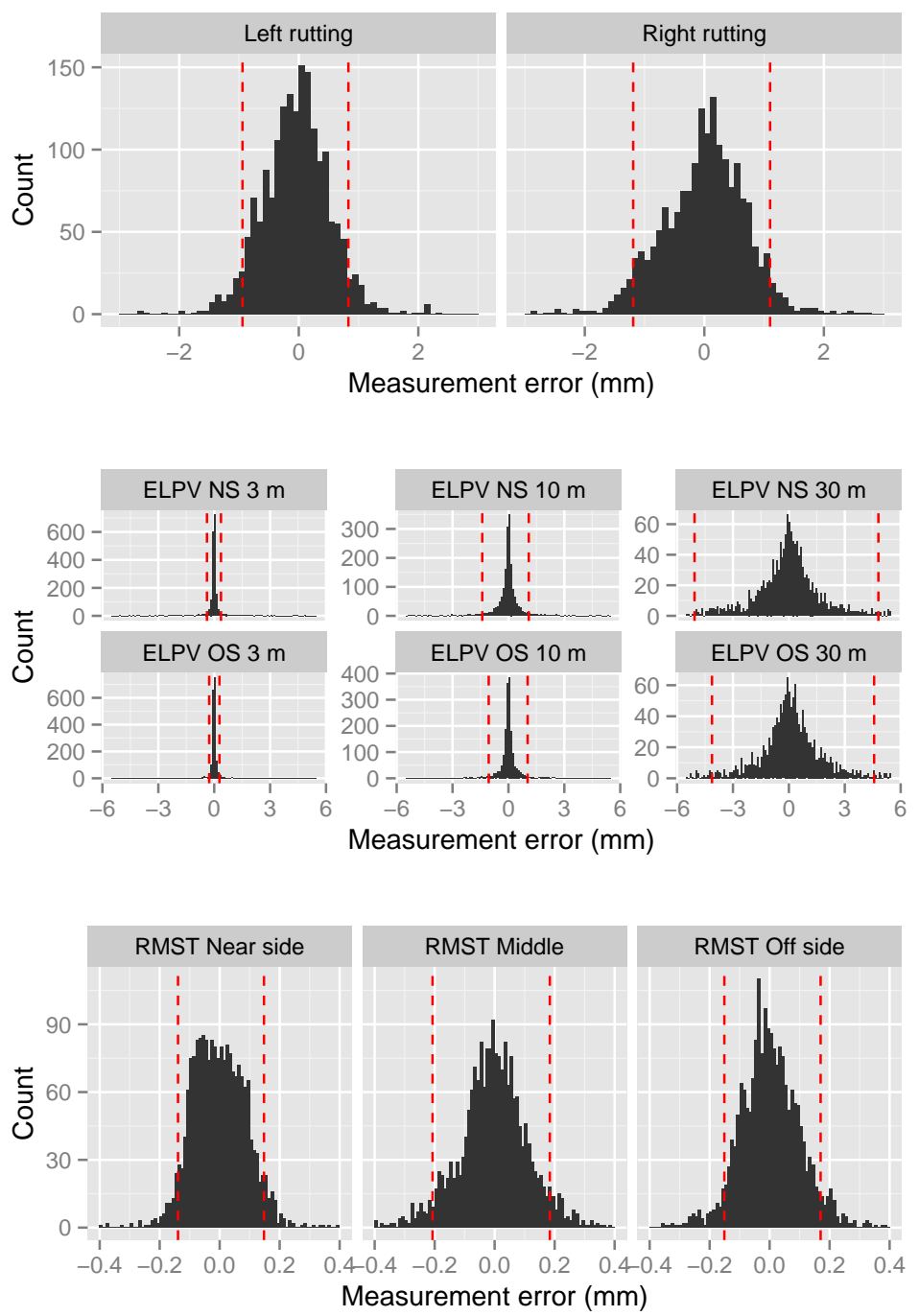

Figure 1: Histograms of measurement errors for all road condition variables. Red dotted lines show the $90^{\text {th }}$ percentile.

\subsection{Multivariate Finite Mixture Models}

Consider the road condition variables as a multivariate random variable $\mathbf{Y}_{i}, i=1, \ldots, N$, where $N$ is the number of 10 meter segments. In order to model the change in surface condition, the variables need to be transformed so that the initial condition does not affect the anlysis. This is done by letting $\mathbf{Y}_{i}^{*}=\mathbf{Y}_{i}-\mathbf{Y}_{\max (0, i-1)}$. Hence the first year of data, 2013, will only be used to create the new variable, which leaves two years worth of actual changes in road condition. If a longer time series were present, a longitudinal model would have been suitable. However, 
because of the short time series $(t=2)$, the estimation of any within-subject variation will be unstable. Also, if the change in road condition is assumed to be an $\operatorname{AR}(1)$-process - that is; having a memory of one lag - the difference between measurement occasions is independent and identically distributed (i.i.d). This assumption cannot be verified with such a short time series as only two realisations, but with the data at hand it is a reasonable approach. Therefore we choose to fit multivariate gaussian mixture models, assuming that the change in road condition between the two measurement occasions is a stationary i.i.d. process.

Following the structure used by Fraley and Raftery (2002), the likelihood for a multivariate mixture given data $\mathbf{y}=\mathbf{y}_{i}, \ldots, \mathbf{y}_{n}$ with $K$ components is:

$$
L_{m i x}\left(\theta_{1}, \ldots, \theta_{K} ; w_{1}, \ldots, w_{K} \mid \mathbf{y}\right)=\prod_{i=1}^{n} \sum_{j=1}^{K} w_{j} f_{j}\left(\mathbf{y}_{i} \mid \theta_{j}\right)
$$

where $f_{j}$ and $\theta_{j}$ are the density and parameters of the $j$ th component in the mixture, and $w_{j}$ is the probability that an observation belongs to the $j$ th component $\left(w_{j}>0\right.$ and $\left.\sum_{j=1}^{K} w_{j}=1\right)$. For a gaussian mixture, $f_{j}$ is the multivariate normal density $\phi_{j}$ parameterized by its mean $\mu_{j}$ and and covariance matrix $\Sigma_{j}$ :

$$
\phi_{j}\left(\mathbf{y} \mid \mu_{j}, \Sigma_{j}\right) \equiv \frac{\exp \left\{-\frac{1}{2}\left(\mathbf{y}_{\mathbf{i}}-\mu_{j}\right)^{T} \Sigma_{j}^{-1}\left(\mathbf{y}_{\mathbf{i}}-\mu_{j}\right)\right\}}{\left.\sqrt{\operatorname{det}\left(2 \pi \Sigma_{j}\right.}\right)}
$$

Fraley and Raftery (2002) describe the clusters as being centered around the mean $\mu_{j}$ with the covariance matrix $\Sigma_{j}$ determining the shape, volume and orientation of the clusters. If $\Sigma_{j}=\lambda I$, giving spherical clusters of the same size, only one parameter is needed to characterize the covariance structure. For the most relaxed type of covariance structure implying an unrestricted $\Sigma_{j}, K(d(d+1) / 2)$ parameters are required for d-dimensional data. A general framework for the geometric properties of the covariance matrix was proposed by Banfield and Raftery (1993), which is a decomposition on the form:

$$
\Sigma_{j}=\lambda_{j} D_{j} A_{j} D_{j}^{T}
$$

where $D_{j}$ is the orthogonal matrix of eigenvectors, $A_{j}$ is a diagonal matrix with elements proportional to the eigenvalues, and $\lambda_{j}$ is a constant of proportionality. This composition is used by Fraley and Raftery (2002) to put constrains on the cluster covariance matrix and evaluate which structure is most appropriate for the data. $D_{j}$ governs the orientation of the $j$ th component of the mixture; $A_{j}$ governs the shape and $\lambda_{j}$ the volume.

The actual clustering is performed with model-based agglomerative hierarchical clustering as described by Fraley and Raftery (2002), in which the approximate classification likelihood is computed as:

$$
L_{C L}\left(\theta_{1}, \ldots, \theta_{K} ; \ell_{1}, \ldots, \ell_{n} \mid \mathbf{y}\right)=\prod_{i=1}^{n} f_{\ell_{i}}\left(\mathbf{y}_{i} \mid \theta_{\ell_{i}}\right)
$$

where $\ell_{i}$ are the labels indicating the classification of each observation, i.e. $\ell_{i}=j$ if $\mathbf{y}_{i}$ belongs to the $j$ th component. 


\subsection{Model Selection}

Using the R-package mclust written by Fraley and Raftery (2002), a two component mixture model $(K=2)$ is fitted to the $d=11$ dimensional road condition measurement data set using the EM algorithm (Dempster et al., 1977; McLachlan and Krishnan, 2007). The deterministic choice of the number of components is motivated by the nature of the data. The segments belonging to the unchanged group is likely to have a very narrow distribution with small variances, while the segments belonging to the change group will have a wide distribution with larger variances. The mean is not necessarily different between the two groups, because not all variables although measuring different aspects of road condition are likely to change at once.

There is always a possibility of identifying more clusters than what is theoretically sound. The Bayesian Information Criterion (BIC) (Schwartz, 1978) is used to evaulate the goodness of fit of several models with up to six clusters and different covariance structures. The overall highest BIC (-83125.37) was found for a model with three clusters and a covariance matrix with varying orientation, shape and volume. A three cluster model would have been useful if the road condition variables were all changing in the same fashion, with deterioration showing as a positive change and maintenance showing as negative change. However, this is not the case for all of the variables. RMST can have a negative change due to both maintenance and deterioration. In a three cluster mixture model, the segments where deterioration is showing in the RMST measurements might be classified into a cluster consisting of segments showing negative change due to maintenance in other variables.

To avoid losing any deterioration information, we will choose a model consisting of a mixture of two distributions where the change group consist of segments showing both positive and negative change. The mixture models with the highest BIC among the two cluster models is the one with the most flexible covariance structure $(\mathrm{BIC}=-111840.38)$. Because of the nature of the two distributions described in the previous paragraph, a flexible structure between clusters in both shape, volume and orientation is theoretically valid.

\section{Results}

\subsection{Clustering results}

Of the 8824 road segments, 6136 are classified into the unchanged group and 2688 into the change group. Within the change group, 1663 of the segments had measurements which indicated change both between 2013 and 2014 and between 2014 and 2015. 1025 segments had measurements which indicated a change in road condition only between 2013 and 2014.

Table 2 shows the parameter estimation results for both groups. Road condition is expected to be stationary, and mean values are around zero for all variables except ELPV 30 meter. The change group generally have mean values towards the negative side, indicating that the effect of maintenance is higher in most variables than the effect of deterioration. Variances for the variables in the change group are 5.7 up to 330 times larger than variances in the unchanged group, which is in line with the assumption of an underlying outlier distribution. 
Table 2: Parameter estimation results

\begin{tabular}{|l|cc|cc|}
\hline & \multicolumn{2}{|c|}{ Unchanged group } & \multicolumn{2}{c|}{ Change group } \\
& $w_{1}=0.76$ & \multicolumn{2}{c|}{$w_{2}=0.24$} \\
\hline Parameter & $\mu_{1}$ & $\sigma_{1}^{2}$ & $\mu_{2}$ & $\sigma_{2}^{2}$ \\
\hline Left rutting & 0.35 & 0.38 & -0.66 & 8.34 \\
Right rutting & 0.16 & 0.23 & -1.32 & 10.51 \\
ELPV 3 m NS & -0.007 & 0.002 & -0.009 & 0.66 \\
ELPV 3 m OS & -0.006 & 0.002 & -0.05 & 0.53 \\
ELPV 10 m NS & -0.08 & 0.034 & -0.22 & 4.39 \\
ELPV 10 m OS & -0.08 & 0.034 & -0.36 & 5.05 \\
ELPV 30 m NS & -1.98 & 8.37 & -4.39 & 97.3 \\
ELPV 30 m OS & -1.97 & 8.76 & -4.42 & 50.0 \\
RMST Mean Middle & 0.005 & 0.006 & -0.04 & 0.054 \\
RMST Mean NS & 0.02 & 0.008 & -0.15 & 0.072 \\
RMST Mean OS & 0.001 & 0.008 & -0.07 & 0.036 \\
\hline
\end{tabular}

Left and right rutting both have long negative tails for the change group, indicating that rutting had improved for a lot of segments between 2013 and 2015 - i.e. these segments have been maintained since deterioration in rutting will always result in positive change. The distribution of the unchanged group matches the findings from the investigation of the measurement errors (Figure 1). 90 percent of the observations in the unchanged group is within the interval $[-0.95$ : $1.58]$ for left rutting and [-1.24:0.39] for right rutting. The longer positive tail of the change group distribution is thought of as the deterioration in two years time, or measurement/recording errors that are more severe than expected.

ELPV $3 \mathrm{~m}$ and $10 \mathrm{~m}$ for off side mesurements are shown in middle panel of Figure 2. The unchanged group has a slightly more narrow interval than the measurement errors in Figure 1, with 90 percent of the observations in an interval of $[-0.08: 0.06]$ for $3 \mathrm{~m}$ and $[-0.44: 0.15]$ for $10 \mathrm{~m}$ measurements. This is an indication that some of the segment in the change group might actually be measurement errors; or, as the very flat density plots for the ELPV variables indicates, segments which show change in other variables is likely to show small changes in ELPV too.

The RMST variable for the middle part of the road has a thicker tail around -0.6 for the change goup. The tail is slightly shifted to the right for the near side measurments, which has a small peak around -0.3. This result is coherent with how the texture is expected to deteriorate: at first, the road surface is polished (RMST decreases), but later in the deterioration process stones are beginning to come out (RMST increases). The middle part of the road will always be behind the near and off sides in the deterioration process because vehicles drive there less frequently.

All change distribution has a more or less distinct peak around zero. The probabilities for each segment is based on the multivariate response variable $\mathbf{Y}_{i}$, and since the analysis has only three years worth of data, it is likely that not all road condition variables have changed during this time. Some segments will be clustered into the change group although they do not show any change in some variables. 

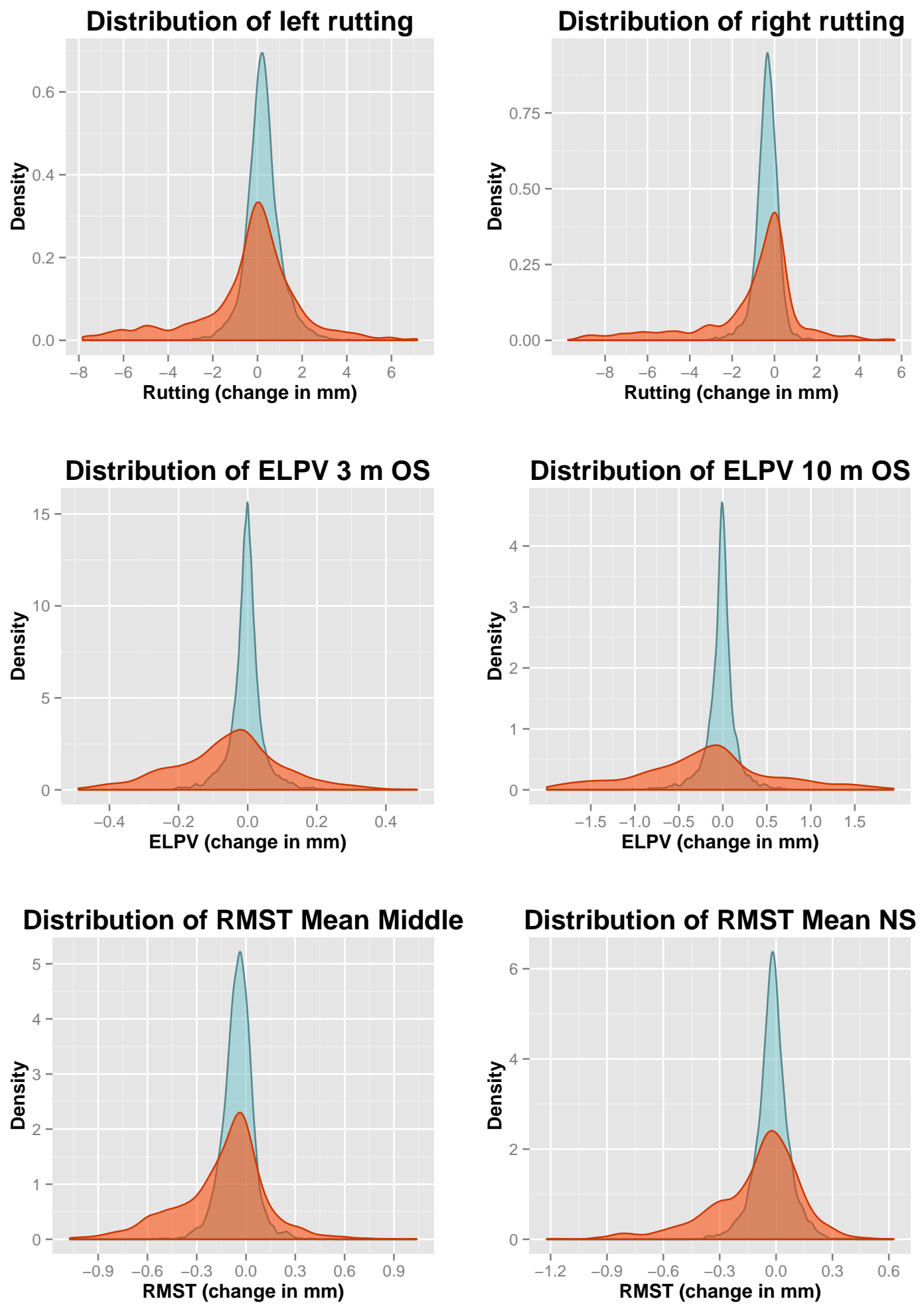

Figure 2: Density plots of the segments divided into the change group (red) and unchanged group (blue). 


\subsection{Mapping clusters}

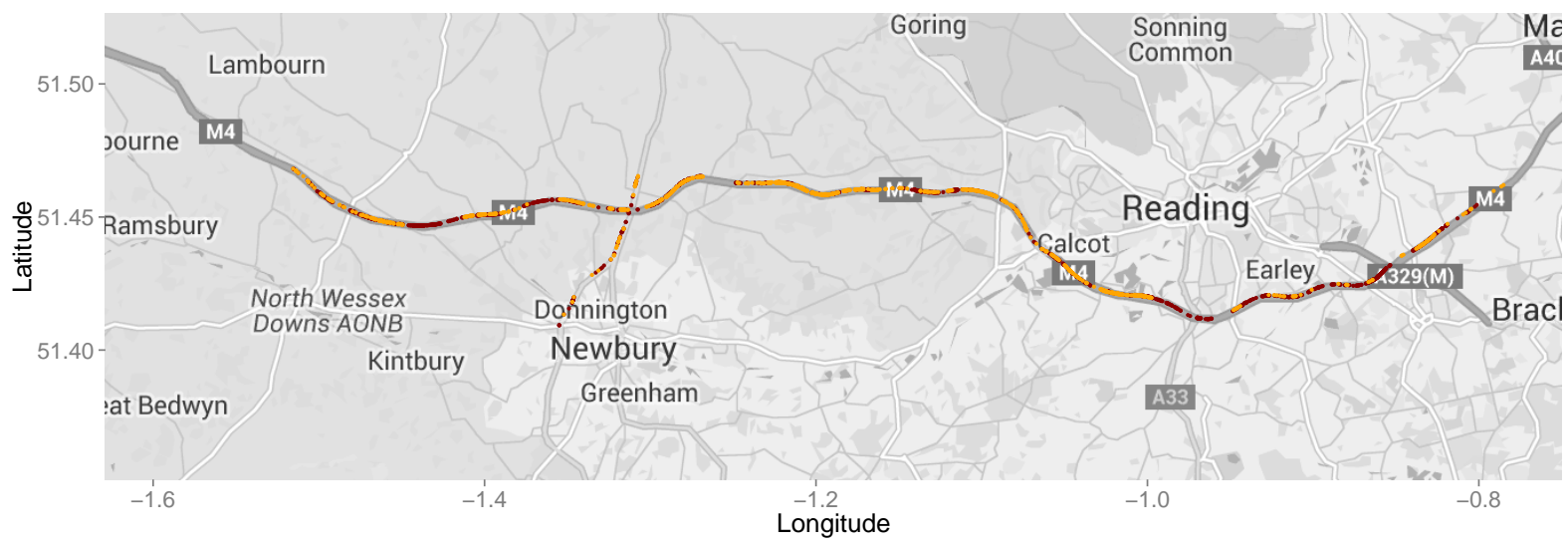

Figure 3: Map of the M4/A34 intersection with yellow and red dots representing segments in the change distribution.

Figure 3 shows the segements that belong to the change group as red or yellow dots. Red dots represent the segments with measurements indicating change both 2013-2014 and 20142015, while yellow dots are segments that only showed change in 2013-2014. No segments were classified into the change group based on change in road condition measurements only between 2014-2015. The change group may include segments that have changed either due to deterioration or maintenace, where maintenance often results in greater changes in road condition. In order to determine if the classification is of any value in practice, data regarding maintenance activities is plotted in the road network (Figure 4).

Figure 4 shows a close up on a roundabout on the M4. Maintenance that occured 2013 and 2014 is drawn as blue lines, and maintenance that occured after January 2015 is drawn as green lines. Because only start and end coordinates of the section ID is available, and not coordinates of the maintained segments, the blue and green lines will cover longer sections than where the maintenance actually was carried out. However, if the maintenance data and the change group segments correlate, there is an indication that the classification can capture real changes.

The red segments beneath the blue line in Figure 4 is likely to have changed because of maintenance. A look at the raw data for these segments shows large negative changes in almost all variables for these segments between 2013 and 2014; e.g. $6-11 \mathrm{~mm}$ for rutting, 0.5-1.5 mm for ELPV $3 \mathrm{~m}$ and $0.6-1.5 \mathrm{~mm}$ for RMST, which are way outside the measurement error interval. The data from 2015 for these segments is missing.

The green lines represent maintenance acitvities that took place after the measurements of the road condition were performed. That is, the red segments beneath the green lines have not changed because of previous maintenance; they were maintained shortly after the road condition was surveyed in January 2015. The fact that they were maintained so soon is a sign of change due to deterioration. Previous research (source) shows that deterioration is often constant for a certain amount of time, and then has an exponential rather than linear development. If a segment is identified as a candidate object due to a bad condition, it has often shown an increasing deterioration pattern. Looking at the raw data from these segments, there are positive changes in the range of $2-5 \mathrm{~mm}$ for rutting, a strong indication of deterioration. The segments also show negative changes for RMST between $0.3-0.8 \mathrm{~mm}$, which is slightly outside the expected 
measurement error interval. Negative change can imply both maintenance and deterioration for RMST. However, maintenance is expected to yield larger changes than deterioration which is the case in this example. ELPV for these segments have only small changes within the expected measuement error interval.

The example in Figure 4 indicates that both changes due to maintenance and changes due to deterioration can be captured by the finite mixture model.

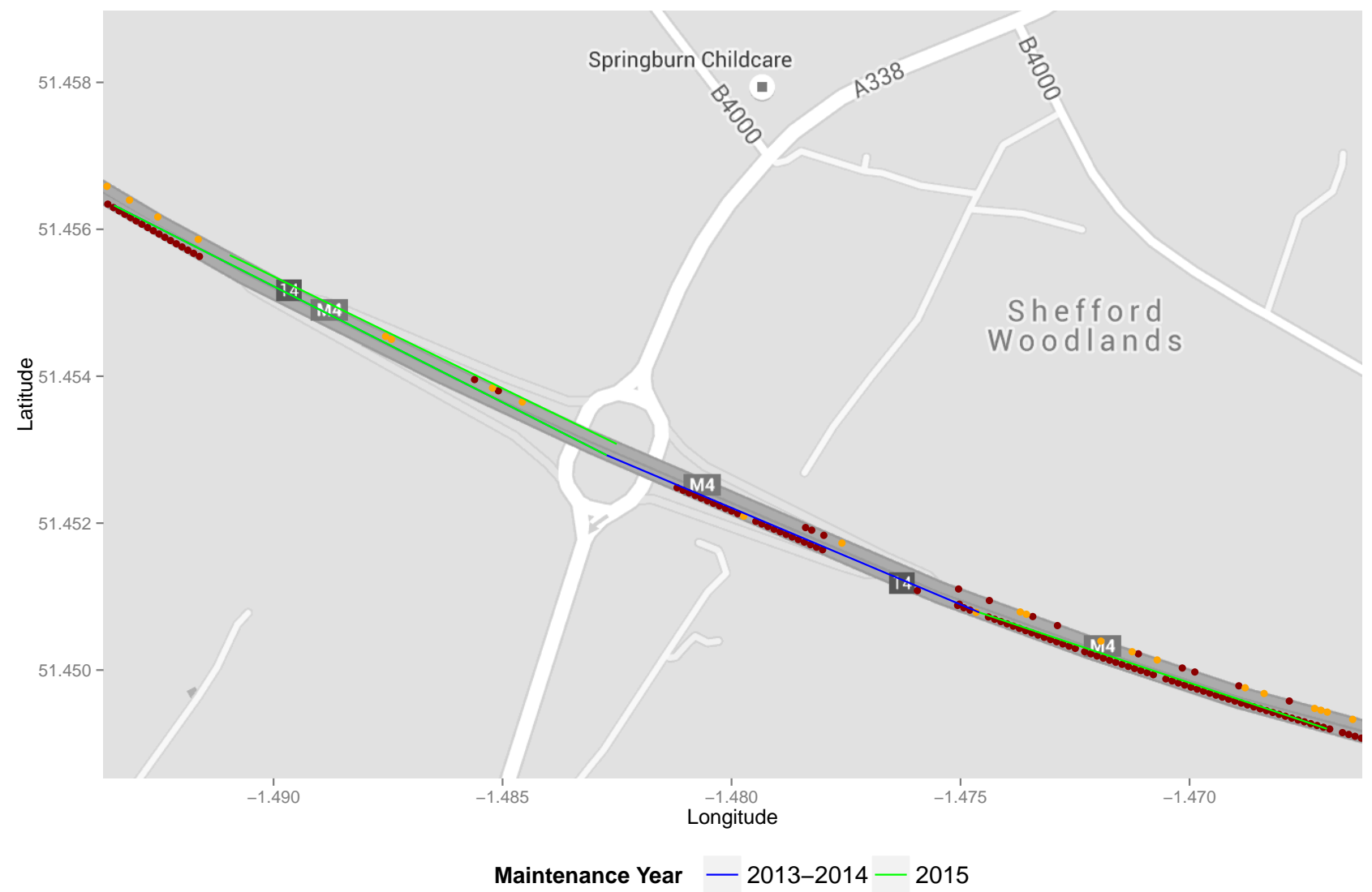

Figure 4: Map of a part of the M4 highway. The left most lane in each direction is used in the analysis.

\section{Discussion}

With only three years worth of data, the depencency structure between road condition measurements cannot be evaluated. It is assumed in this paper that they can be represented by 
an AR(1)-process, thus implying that the first difference of measurements is a stationary IIDprocess. With measurements from more years available, it would be possible to check this assumption, and if it does not hold, a longitudinal model which takes within-subject correlation into account may be used.

The mean vector $\mu_{2}$ is negative for all road condition variables in the change group. The result is expected because changes in road condition due to maintenance activities (which can account for most, but not all, negative change) are likely to be greater than changes due to deterioration. However, negative changes in road condition might also be a sign of deterioration for some variables; in this model that may be the case of RMST. If it was certain that all variables showed positive change due to deterioration and negative change due to maintenance, a mixture of three distributions would be worth considering - change due to deterioration and/or large positive measurement errors, unchanged, change due to maintenance and/or large negative measurement errors. However, because negative change in RMST can occur due to both maintenance and deterioration this variable could not be included in such a model. RMST is one of the more sensitive variables (in the sense that it is possible to find real changes over such a short time scale as 1-2 years) and therefore it would be unwise to disregard it. When the mixture models consist of two distributions, defined as a change and an unchanged group, complementary data from maintenance records is necessary to distinguish between change due to maintenance or deterioration.

The findings of this study also reveal another possible use of the finite mixture models: identifying maintenance activities. There is often missing data in pavement management databases and the results from the finite mixture model may also be useful in order to find segments with missing maintenance records. A way of doing this is to look at e.g. the development of rutting for segments in the change group, where negative change (i.e. less rutting) in road condition on several adjacent segments is very likely to occur because of maintenance.

If maintenance data with coordinates is available, it is straight forward to identify segments that have changed because of previous maintenance activities. For the data used this paper, only start and end coordinates of a section (consisting of several $10 \mathrm{~m}$ segments) is provided which limits the precision. Apart from maintenance and deterioration, it is also possible that large measurement errors is identified as change. The finite mixture model is not an automatic deterioration detection, but rather a way to identify segments that deviate from the average deterioration pattern which is considered to be fairly stationary. As a future research topic, combining predictive pavement deterioration models with finite mixture models could be a way of further explore the possibilities of finding early deterioration.

\section{Conclusion}

The finite mixture model was able to classify segments into a change group and an unchanged group, where the distribution of the segments in the unchanged group is similar to the distribution of the measurement errors. The segments in the change group were compared with data from maintenance records. It could be concluded that the change group most likely consists of segments that have changed due to maintenance activities and deterioration, because they matched locations of both previous and (at the time of the measurement occasion) future maintenance. Finite mixture models can be a helpful tool to identify segments where the road condition measurements is deviating from the stationary state of a road surface that is not maintained or deteriorating quickly. Identifying such segments can be helpful in order to reduce the number of possible maintenance candidates when resources is limited, and thus make maintenance desicions more effective. However, it is not possible to fully distinguish between change due to maintenance, deterioration or severe measurement errors using the mixture models approach 
without further investigation of the data. 


\section{References}

Jeffrey D. Banfield and Adrian E. Raftery. Model-based gaussian and non-gaussian clustering. Biometrics, 49:803821, 1993.

Kim Daeyoung and Bruce G. Lindsay. Empirical identifiability in finite mixture models. Annals of the Institute of Statistical Mathematics, 67(4):745-772, 2015.

Daniel Defays. An efficient algorithm for a complete link method. The Computer Journal, 20 (4):364-366, 1977.

Arthur P Dempster, Nan M Laird, and Donald B Rubin. Maximum likelihood from incomplete data via the em algorithm. Journal of the royal statistical society. Series B (methodological), pages $1-38,1977$.

Chris Fraley and Adrian E. Raftery. Model-based clustering, discriminant analysis and density estimation. Journal of the American Statistical Association, 97:611-631, 2002.

J. A. Hartigan and M. A. Wong. Algorithm as 136: A k-means clustering algorithm. Journal of the Royal Statistical Society. Series C, 28(1):100-108, 1979.

Feng Hong and Jorge A. Prozzi. Estimation of pavement performance deterioration using bayesian approach. Journal of Infrastructure Systems, 12(2):77-86, 2006. doi: 10.1061/ 1076-0342.

Thomas Laitila and Maria Karlsson. Finite mixture modeling of censored regression models. Statistical Papers, 55(3):627-642, 2014.

Si Quang Lee, Nicolas Lartillot, and Olivier Gascuel. Phylogenetic mixture models for proteins. Philosophical Transactions of the Royal Society B: Biological Sciences, 363(1512):3965-3976, 2008.

Nam Lethanh and Bryan T. Adey. Use of exponential hidden markov models for modelling pavement deterioration. International Journal of Pavement Engineering, 14(7):645-654, 2012. doi: $10.1080 / 10298436.2012 .715647$.

Geoffrey McLachlan and Thriyambakam Krishnan. The EM algorithm and extensions, volume 382. John Wiley \& Sons, 2007.

Geoffrey McLachlan and David Peel. Finite Mixture Models. John Wiley \& Sons, New York, 2000.

Geoffrey J. McLachlan and Kaye E. Basford. Mixture Models. Inference and applications to clustering. Dekker, New York, 1988.

Mark Pagel and Andrew Meade. A phylogenetic mixture model for detecting patternheterogeneity in gene sequence or character-state data. Systematic Biology, 53(4):571-581, 2004 .

Byung-Jung Park and Dominique Lord. Application of finite mixture models for vehicle crash data. Accident Analysis \& Prevention, 14(4):683-691, 2009. doi: 10.1016/2009.03.007.

G. Schwartz. Estimating the dimenson of a model. The Annals of Statistics, 6:461-464, 1978.

Robin Sibson. Slink: an optimally efficient algorithm for the single-link cluster method. The Computer Journal, 16(1):30-34, 1973. 
Hugo Steinhaus. Sur la division des corp materiels en parties. Bull. Acad. Polon. Sci, 1:801-804, 1956.

Joe H Ward Jr. Hierarchical grouping to optimize an objective function. Journal of the American statistical association, 58(301):236-244, 1963. 